Review Article

\title{
Management of androgenetic alopecia: a review
}

\author{
Deepa Dev Chhetri, Xin Huang*
}

Department of Dermatology, Shanghai Tongji Hospital Affiliated to Tongji University, Shanghai, China

Received: 21 November 2020

Revised: 14 December 2020

Accepted: 23 December 2020

\section{*Correspondence:}

Dr. Xin Huang,

E-mail: alida_huang@163.com

Copyright: (C) the author(s), publisher and licensee Medip Academy. This is an open-access article distributed under the terms of the Creative Commons Attribution Non-Commercial License, which permits unrestricted non-commercial use, distribution, and reproduction in any medium, provided the original work is properly cited.

\begin{abstract}
Androgenetic alopecia is a patterned hair loss disorder affecting approximately $73 \%$ of men and $57 \%$ of women in their lifetime, this affection can cause psychological effects like low self-stem and depression diminishing the quality of life. Currently, finasteride and minoxidil are the only two drugs approved by the food and drug administration (FDA), and devices of low-level laser therapy are FDA cleared. Alternative therapies for androgenetic alopecia treatment include a drug like dutasteride, botulinum toxin A, cell-based therapies like adipose-derived stem cellconditioned medium and platelet-rich plasma protocols. A combination of two or more therapies can be found in the literature, the general consent indicates that any combination has higher efficiency than a single therapy. Finasteride is in less use due to the related sexual side effects, some adjuvant techniques have emerged to improve the delivery and effectiveness of minoxidil, i.e. micro needling. The surgical approach is mainly focused on hair transplant surgery, which offers relatively less invasive procedures and is a more suitable option for long time results, however, it is expensive. Recently, drug-assisted delivery techniques have emerged to improve the efficiency of conventional drugs; transdermal drug delivery through ultrasound pressure waves means creating diffusion channels, increasing skin permeability and stimulating cell differentiation and growth factor utilization along with minoxidil absorption. Another approach for drug-assisted delivery is the embodiment of finasteride in polymer-based microspheres aiming for a sustained and controlled delivery, which can be beneficial to reduce the regular doses decreasing adverse effects.
\end{abstract}

Keywords: Androgenetic alopecia, Hair loss, Finasteride, Minoxidil, Alternative therapies

\section{INTRODUCTION}

Androgenetic alopecia (AGA) also known as hair loss pattern baldness is a common condition affecting not only men but women, causing hair loss in well-defined patterns. This genetic condition affects $58 \%$ of men and $21 \%$ of women, and its effects increase with age. ${ }^{1}$ The main cause related to androgenetic alopecia is the abundance of dihydrotestosterone (DHT), a strong form of testosterone produced by the action of the two types (I and II) of the enzyme $5 \alpha$-reductase, DHT cause miniaturization of the follicles leading to hair transformation into vellus hai. ${ }^{2}$ AGA can also cause psychological distress leading to low self-stem and depression, making people have a low-quality life, androgenetic alopecia affects around 80 million people just in the United States. ${ }^{3-5}$ Due to the importance of this disorder, the research for therapeutic options to prevent hair loss caused by androgenetic alopecia has derived in a wide variety of treatments of different nature from oral drugs to surgical approaches.

The most used treatments for AGA include oral drugs like finasteride and topical administration of minoxidil, however, the effectiveness of these conventional therapies is low. In recent years, new therapies have become available including phototherapy techniques like low-level laser therapy (LLLT), cell-based therapies like cell stem therapy (platelet-rich plasma, adipose-derived 
stromal vascular cells, and human follicle mesenchymal stem cells), and surgical approaches like hair transplant and hairline-lowering surgeries. Furthermore, alternative therapies using combinations of techniques have raised to improve the delivery and bioavailability of minoxidil, for instance, oral administration of minoxidil, Lowfrequency dual-frequency ultrasound-mediated microbubble cavitation for transdermal minoxidil delivery and micro needling followed by topical administration of minoxidil.

This review aims to provide an update of the current status of the emergent alternative therapies to prevent hair loss caused by androgenetic alopecia, in this way dermatologists can manage treatments for their patients considering each particular case. The literature research for this review was made including relevant studies for each treatment/technique, in further sections therapies will be described and addressed according to their working principle.

\section{DRUG THERAPIES}

Finasteride was the first drug approved by the FDA for androgenetic alopecia treatment in 1997, an inhibitor of type II $5 \alpha$-reductase enzyme responsible for the conversion of testosterone in dihydrotestosterone. ${ }^{6}$ DHT is a strong androgen that induces follicle miniaturization and decreases the hair growth rate, which appears to be the main cause of AGA. Since its approval, the use of this drug raised concerns regarding sexual adverse effects (decrease in libido and erectile dysfunction), despite all the controversies around it, finasteride has shown the effectiveness of up to $60 \% .^{7}$ The dose recommended for patients of each gender is $1 \mathrm{mg} /$ day for men and 5 $\mathrm{mg}$ /day for women, a recent study showed that this treatment should be intended for women in the pre and post-menopausal stage were proved to have more efficiency. ${ }^{8}$

Dutasteride is an inhibitor of the two types of $5 \alpha$ reductase, this drug has been used for AGA treatment since 2009. ${ }^{9}$ As an inhibitor of the two types of the enzyme, a better result would be expected compared to finasteride. A comparison study of finasteride versus dutasteride at different doses was conducted by Gubelin Harcha et al they found that similar results were obtained for $1 \mathrm{mg} /$ day of finasteride and $0.2 \mathrm{mg} /$ day of dutasteride, however, at a higher dose of dutasteride $(0.5 \mathrm{mg} /$ day $)$ a significant increase in hair count was obtained in comparison to regular treatment of finasteride. ${ }^{10}$ Although no sexual adverse effects are claimed by different authors, the same concerns as those found for finasteride are related to the dutasteride treatment.

Minoxidil is the only topical drug approved by the FDA for androgenetic alopecia treatment, the effect of this drug seems to have several contributions in the biological response; increases blood flow, increases the mitosis of hair matrix keratinocytes and promotes growth hair faster and thicker; prolongs the anagen phase, and stimulates the kenogen follicles to initiate a new hair growth cycle. $^{3,11}$ Typical minoxidil treatment consists in topical application of $2-5 \%$ solution, showing better results with the application of $5 \%$ solution twice a day for men while having similar results in women using both solution concentrations (recommended dose for women is topical application of $2 \%$ solution once a day). ${ }^{12}$ In new studies, the oral administration of minoxidil was proposed as an alternative, showing similar results than those obtained for topical administration in men, however, side effects like hypertrichosis $(93 \%)$ and pedal edema (10\%) can appear even at doses as low as $0.25 \mathrm{mg} /$ day. ${ }^{13,14}$

A more recent approach consists in a combination of oral administration of finasteride (1 mg/day) along with a topical application of 5\% minoxidil solution (6), although the drug combination has shown better results than either of the two drugs alone, more research is needed to determine the most suitable doses to avoid side effects as much as possible. More complex treatments like that reported by Tanaka et al using different ways of administration and combination of minoxidil and finasteride seem to show good results aiming only for Asian men, however, stronger evidence is needed to determine the reliability of this study. ${ }^{15}$

\section{PHOTOTHERAPY}

The low-level laser therapy (LLLT) is a technique used to generate an overall effect in the human body called photo biomodulation (PBM), this therapy is also known with the names of low-level light therapy, red light therapy, and soft laser. PBM is the effect caused by low laser beams, which improve wound healing and stimulate hair growth. ${ }^{16}$ Although the mechanism of action of LLLT is not clear, it has been gaining popularity and recognition as a suitable treatment for AGA, in fact, there are several FDA-cleared devices using low-level laser therapy. ${ }^{17}$ Some of the effects of this phototherapy are accelerated mitosis of keratinocytes and fibroblast, inhibition of nitric oxide production by the cytochrome $\mathrm{C}$ oxidase and decrease of inflammation. ${ }^{17}$

Despite its increased popularity, there are no wellestablished parameters of the low-level laser therapy for the AGA treatment, the wavelengths commonly used range from 500 to $1100 \mathrm{~nm}$, the power intensity can vary from 3 to $90 \mathrm{~mW} / \mathrm{cm} 2$ and the fluency from 1 to 4 $\mathrm{J} / \mathrm{cm}^{2} .{ }^{18}$ Some authors reported good results in a narrower wavelength range from 650 to $780 \mathrm{~nm}$, using a daily treatment in both men and women. ${ }^{17}$

\section{CELL-BASED THERAPIES}

Stem cell therapy (SCT) is one of the more recent and promising approaches to treat androgenetic alopecia. SCT uses exogenous cells, clinical trials can use both allogenic and autologous cell sources. Adult stem cells can be harvested from different tissues including fat, scalp, bone 
marrow and peripheral blood. ${ }^{19}$ Bone marrow stem cells (BMSC) have been used for its relatively easy and fast harvesting, however, the procedure to obtain them is invasive and causes pain to the donor, also the low yields of cell detachment conventions make BMSC not the best option for hair loss treatment.

Owing to the constraints of the use of BMSC, the adipose-derived stem cells have been proposed due to their ability to differentiate into mesenchymal lineage cells and the release of several growth factors. ${ }^{20}$ Fat tissue is harvested in a less invasive way (i.e. liposuction) and in more abundant quantities than other stem cell tissues. Adipose-derived stem cell-conditioned medium contains growth factors such as vascular endothelial growth factor, hepatocyte growth factor, platelet-derived growth factor, and insulin-like growth factor $1 .^{21}$

A recent study showed the use of Adipose-derived stem cell-conditioned medium as a treatment for hair loss, the treatment consisted of an intradermal injection of the medium every 3-5 weeks in a 6-session experiment. The results showed a significant increase in the hair count in both men and women $(29 \pm 4.1$ in men and 15.6 \pm 4.2 in women) being more effective for men. ${ }^{22}$

Platelet-rich plasma (PRP) is a cell-based therapy consisting of an autologous preparation of plasma with concentrated platelets with a concentration of around 94\%. The use of PRP for hair growth is based on the content of growth factors in platelets. Platelet-rich plasma contains six fundamental growth factors; fibroblast growth factor (b-FGF), platelet-derived growth factor (PDGF), vascular endothelial growth factor (VEGF), epidermal growth factor (EGF), transforming growth factor-(TGF-), and insulin-like growth factor-1 (IGF-1). ${ }^{19}$ Growth factors found in platelet-rich plasma can increase hair regrowth by binding to their receptors expressed by stem cells of the follicle bulge region, then stem cells favor the proliferative phase of the hair follicle, producing the anagen follicular unit and enhance hair regrowth.

Platelet-rich plasma protocols can vary widely, and the use of each should be evaluated by the physician to determine its suitability in every patient. The different types of PRP rely on their cells content and fibrin design: 1) leukocyte-poor PRP (LP-PRP) 2) PRP and leukocyte (L-PRP) 3) leukocyte-poor platelet-rich fibrin (LP-PRF) 4) leukocytes and platelet-rich fibrin (L-PRF)

Each type of PRP can be obtained upon changes in preparation protocol. Anitua et al., performed a study using Plasma Rich in Growth Factors (PRGF) for the treatment of androgenetic alopecia. The results showed a statistically significant increase in hair density count from $117 \pm 29$ follicles $/ \mathrm{cm}^{2}$ to $156 \pm 36$ follicles $/ \mathrm{cm}^{2}$ after therapy was applied. Although positive results like those described here have been obtained for PRP therapy, there are no standardized protocols, therefore more research is needed.

\section{MICRONEEDLING}

The microneedling procedure consists of making small holes in the scalp with a dermaroller containing titanium needles until mild erythema is reached, the preferred needle size used for AGA treatment is $1.5 \mathrm{~mm}$ in length. In worthy to mention that microneedling is considered an adjuvant technique, this implies that its application has as a goal to help improve the effectiveness of the main treatment (i.e. topical minoxidil administration). Microneedling act through the stimulation of the stem cells and activation of growth factors, which also stimulate the dermal papillae. ${ }^{23}$ In recent work, Kumar et al performed a comparative study of $5 \%$ topical minoxidil plus microneedling (once a week) and minoxidil alone. The results of this study indicate that the topical minoxidil plus microneedling treatment has a significant effect on hair growth, the patients reported up to $50 \%$ improvement versus the treatment with minoxidil alone. ${ }^{24}$

Microneedling has also been used along with PRP+topical minoxidil and compared to the topical minoxidil treatment alone, the results in such a study showed significant improvement in the patients in the group receiving the microneedling $+\mathrm{PRP}+5 \%$ minoxidil versus the patients under the minoxidil alone. ${ }^{25}$ Another study reported the comparison of microneedling $+5 \%$ minoxidil treatment against microneedling+PRP, in that case, both treatments showed similar results been both effective in AGA treatment. ${ }^{26}$

\section{BOTULINUM TOXIN A}

Botulinum toxins are a group of neurotoxins produced by the bacteria Clostridium botulinum, consisting of eight different toxins (A, B, C1, C2, D, E, F, and G). Toxin type $A$ is the strongest toxin of them, and have found a broad kind of applications in medicine. A few studies have been conducted using botulinum toxin $\mathrm{A}$ as an alternative treatment for androgenetic alopecia. The mechanism of action seems to be related to the relaxation of the muscles in the scalp generating an increase in the blood flow. ${ }^{27}$

In a recent study, Liu-Tao et al compared the treatment of finasteride ( $1 \mathrm{mg} /$ day) and application of botulinum toxin $\mathrm{A}$ in a 6-month trial. They concluded that the treatment with the toxin has a similar result as the oral drug against the control patients. The advantage of the new treatment over the finasteride is that no side effects were found. ${ }^{28}$ The Botulinum toxin treatment consists of the direct injection of the neurotoxin direct to the scalp, the doses used in the studies range from 50 to $150 \mathrm{U}$, showing better results for higher doses. ${ }^{27-29}$ Although high efficiency (from 45 to $90 \%$ of the cases showed improvement) was found for this treatment, further study is needed in a well-designed experiment. 


\section{SURGICAL PROCEDURES}

Despite the variety of available treatments for androgenetic alopecia some patients do not respond to most of the previously mentioned therapies, those patients are candidates for some type of surgical treatment. Surgical procedures are very common nowadays, it seems to be a more reliable option for younger men looking for a safer procedure and better esthetical outcome. The surgical approach can involve scalp reduction surgery, hair transplantation or a combination of both. Since hair transplants emerged little application has left for scalp reduction surgery (also known as alopecia reduction), the main disadvantages related to this procedure is its high invasive nature and the low ratio risk-benefit. Scalp reduction surgery is limited to patients with cicatricial alopecia where local flaps can be made without having a secondary defect to restore..$^{30}$

Hair transplants are by far the most used surgical method for hair loss management. The two more accepted techniques for hair transplants are the follicular unit transplantation (FUT) (also known as the strip technique) and the follicular unit extraction (FUE). FUT technique requires the excision of hair-bearing strips from a donor and dissecting into small follicular units. The FUE technique (also known as FOX procedure, follicular unit separation extraction method, Wood's technique, follicular isolation technique, individual follicular group harvesting) uses small individual follicular grafts extracted through manual or motorized punches. ${ }^{30}$ The FUE technique can offer some advantages over FUT, for example, less scarring, shorter recovery time and have minimal risk of nerve injury.

Hair transplant is considered a good procedure to restore hair growth in the long term, however, it is less used than other techniques due to its high cost. Also, requires a high degree in training and surgical skills. Although it is a well-established procedure still presents some challenges like the need to create enough quantities of hair from a donor. $^{31}$

\section{EMERGING APPROACH: ASSISTED DRUG DELIVERY}

Transdermal drug delivery through ultrasound pressure waves is a technique used to make the delivery of topical drugs more efficient. Ultrasound is an FDA approved procedure for transdermal delivery. ${ }^{32}$ The mechanism of action hypothesizes that the shock waves modify the structure of the lipids of the stratum corneum to produce diffusion channels that allowed transdermal delivery.

Ultrasound sonication is believed to act by generating skin permeability through acoustic cavitation. The acoustic cavitation effect is referred as the formation and collapse of microbubbles created in an ultrasound field. Dual frequency (or multi-frequency) ultrasound can improve the acoustic cavitation effect with respect to single-frequency ultrasound. Dual-frequency ultrasound involves the combination of low frequency (from 20 to $60 \mathrm{kHz}$ ) and high-frequency waves (from 1 to $3 \mathrm{MHz}$ ). Moreover, dual-frequency ultrasound sonication improves cavitation activity, which may stimulate neural stem/progenitor cell differentiation and growth factor utilization, while not causing any damage. ${ }^{33}$ In a recent study Liao et al, evaluated the assisted minoxidil delivery in mice models showing promising results, this is a new approach and requires further experimentation for safe application in real patients.

In drug treatments like the oral administration of finasteride two disadvantages have been considered: sexual adverse effects and the daily administration for long periods. Drug encapsulation in polymer-based microspheres is an emerging technique for drug delivery that has been used recently to try to minimize the disadvantages associated with oral administration of finasteride. Kim et al performed a study to compare the daily administration of oral finasteride (standard treatment at $1 \mathrm{mg} /$ day) and the subcutaneous injection of finasteride in poly (lactic-co-glycolic acid) microspheres (the microspheres were injected only at the first day of the trial), the experiment was carried out in mice models for 10 weeks. The results indicate that both treatments have a similar effect with a total hair growth of $60 \% .^{34}$ The authors concluded that long-term studies are necessary to determine the right dose and the periodicity of the injections for further experiments in real patients. The sustainable and controlled delivery of the drug will depend on the hydrolysis rate of the polymer, which is related to the nature of each polymer.

\section{CONCLUSION}

Androgenetic alopecia is the most common hair loss disorder affecting both men and women, and its importance has generated a huge variety of treatments and procedures. Up to this date, the only two drugs approved by the FDA for AGA treatment are finasteride and minoxidil. Dutasteride is the only alternative drug, which has proved to show similar and even better results than finasteride, however, no conclusive results have been obtained. Alternative therapies like low-level laser therapy and platelet-rich plasma protocols have shown good results with statistically significant results. Other therapies like adipose-derived stem cell-conditioned medium and botulinum toxin A administration still need more studies in well-designed experiments to show more consistent results.

Surgical approaches are limited to hair transplant procedures, which seem to be a more suitable option for patients not responding to conventional therapies, they tend to be the more expensive and invasive treatments. Combined therapies have emerged due to the necessity to improve conventional drug treatments; in general, the combination of therapies seem to enhance the hair growth 
with respect to single therapies. Also, some adjuvant procedures like micro needling can enhance the effect of known therapies such as minoxidil and platelet-rich plasma protocols. Since finasteride is responsible for undesirable side effects, minoxidil administration orally or topically in combination with other therapies can offer a safer and reliable option for AGA treatment. Interesting assisted delivery techniques like ultrasonic and polymerbased microspheres seems to be worthy of attention due to the results obtained in mice models, these techniques can help to improve the efficiency and decrease the adverse effects of drugs orally and topically administered.

\section{Funding: No funding sources \\ Conflict of interest: None declared \\ Ethical approval: Not required}

\section{REFERENCES}

1. Light AE. Patterned Loss of Hair in Man; Pathogenesis and Prognosis. Ann N Y Acad Sci. 1951;53(3):729-34.

2. Adil A, Godwin M. The effectiveness of treatments for androgenetic alopecia: A systematic review and meta-analysis. J Am Acad Dermatol. 2017;77(1):136-141.

3. Goren A, Naccarato T. Minoxidil in the treatment of androgenetic alopecia. Dermatol Ther. 2018;31(5):1-2.

4. Savin RC. Psychological effects of androgenetic alopecia on women: Comparisons with balding men and with female control subjects. J Am Acad Dermatol. 1993;29(4):568-75.

5. Cash TF. The psychological effects of androgenetic alopecia in men. J Am Acad Dermatol. 1992;26(6):926-31.

6. Hu R, Xu F, Sheng Y, Qi S, Han Y, Miao Y, et al. Combined treatment with oral finasteride and topical minoxidil in male androgenetic alopecia: A randomized and comparative study in Chinese patients. Dermatol Ther. 2015;28(5):303-8.

7. Andy G, John M, Mirna S, Rachita D, Michael K, Maja $\mathrm{K}$, et al. Controversies in the treatment of androgenetic alopecia: The history of finasteride. Dermatol Ther. 2019;32(2):8-10.

8. Oliveira-Soares R, E Silva J, Correia M, André M. Finasteride $5 \mathrm{mg}$ /day treatment of patterned hair loss in normo-androgenetic postmenopausal women. Int J Trichology. 2013;5(1):22-5.

9. Eun HC, Kwon OS, Yeon JH, Shin HS, Kim BY, Ro BI, et al. Efficacy, safety, and tolerability of dutasteride $0.5 \mathrm{mg}$ once daily in male patients with male pattern hair loss: A randomized, double-blind, placebo-controlled, phase III study. J Am Acad Dermatol. 2010;63(2):252-8.

10. Gubelin Harcha W, Barboza Martínez J, Tsai TF, Katsuoka K, Kawashima M, Tsuboi R, et al. A randomized, active- and placebo-controlled study of the efficacy and safety of different doses of dutasteride versus placebo and finasteride in the treatment of male subjects with androgenetic alopecia. J Am Acad Dermatol. 2014;70(3):489-98.

11. Chen L, Zhang J, Wang L, Wang H, Chen B, Chen B. The Efficacy and Safety of Finasteride Combined with Topical Minoxidil for Androgenetic Alopecia: A Systematic Review and Meta-analysis. Aesthetic Plast Surg. 2020;(270).

12. Kanti V, Messenger A, Dobos G, Reygagne P, Finner A, Blumeyer A, et al. Evidence-based (S3) guideline for the treatment of androgenetic alopecia in women and in men - short version. J Eur Acad Dermatol Venereol. 2018;32(1):11-22.

13. Lueangarun $S$, Panchaprateep $R$, Tempark T, Noppakun N. Efficacy and safety of oral minoxidil $5 \mathrm{mg}$ daily during 24-week treatment in male androgenetic alopecia. J Am Acad Dermatol. 2015;72(5):AB113.

14. Jimenez-Cauhe J, Saceda-Corralo D, RodriguesBarata R, Hermosa-Gelbard A, Moreno-Arrones OM, Fernandez-Nieto D, et al. Effectiveness and safety of low-dose oral minoxidil in male androgenetic alopecia. J Am Acad Dermatol. 2019;81(2):648-9.

15. Tanaka Y, Aso T, Ono J, Hosoi R, Kaneko T. Androgenetic alopecia treatment in Asian men. $\mathrm{J}$ Clin Aesthet Dermatol. 2018;11(7):32-5.

16. Hamblin MR. Photobiomodulation or low-level laser therapy. J Biophotonics. 2016;9(11-12):11224.

17. Darwin E, Heyes A, Hirt PA, Wikramanayake TC, Jimenez JJ. Low-level laser therapy for the treatment of androgenic alopecia: a review. Lasers Med Sci. 2018;33(2):425-34.

18. Ghanaat M. Types of hair loss and treatment options, including the novel low-level light therapy and its proposed mechanism. South Med J. 2010;103(9):917-21.

19. Gentile P, Garcovich S. Advances in Regenerative Stem Cell Therapy in. Cells. 2019;8(466):1-21.

20. Fukuoka H, Suga H. Hair Regeneration Treatment Using Adipose-Derived Stem Cell Conditioned Medium: Follow-up With Trichograms. Eplasty. 2015;15:e10.

21. Park BS, Kim WS, Choi JS, Kim HK, Won JH, Ohkubo F, et al. Hair growth stimulated by conditioned medium of adipose-derived stem cells is enhanced by hypoxia: Evidence of increased growth factor secretion. Biomed Res. 2010;31(1):27-34.

22. Fukuoka H, Narita K, Suga H. Hair Regeneration Therapy: Application of Adipose-Derived Stem Cells. Curr Stem Cell Res Ther. 2017;12(7):531-4.

23. Dhurat R, Sukesh M, Avhad G, Dandale A, Pal A, Pund P. A randomized evaluator blinded study of effect of microneedling in androgenetic alopecia: A pilot study. Int J Trichology. 2013;5(1):6-11.

24. Kumar M, Inamadar A, Palit A. A randomized controlled, single-observer blinded study to determine the efficacy of topical minoxidil plus microneedling versus topical minoxidil alone in the 
treatment of androgenetic alopecia. J Cutan Aesthet Surg. 2018;11(4):211-6.

25. Shah KB, Shah AN, Solanki RB, Raval RC. A comparative study of microneedling with plateletrich plasma plus topical minoxidil (5\%) and topical minoxidil (5\%) alone in androgenetic alopecia. Int $\mathbf{J}$ Trichology. 2017;9(1):14-8.

26. Herakal CK, Vallabhbhai PP, Siddalingappa K. Microneedling with platelet-rich plasma versus microneedling with topical 5\% minoxidil in patients with androgenetic alopecia- a comparative study. J Evol Med Dent Sci. 2017;6(26):2182-6.

27. Singh S, Neema S, Vasudevan B. A pilot study to evaluate effectiveness of botulinum toxin in treatment of androgenetic alopecia in males. J Cutan Aesthet Surg. 2017;10(3):163.

28. Tao L, Zhi-wu L, Jie-sheng D, Xiu-qin D. Local Injections of Botulinum Toxin A in Treatment of Androgenetic Alopecia. J Sun Yat-Sen Univ Sci. 2018;39(3):467-71.

29. 2Zhang L, Yu Q, Wang Y, Ma Y, Shi Y, Li X. A small dose of botulinum toxin $\mathrm{A}$ is effective for treating androgenetic alopecia in Chinese patients. Dermatol Ther. 2019;32(4).
30. Sharma R, Ranjan A. Follicular Unit Extraction (FUE) Hair Transplant: Curves Ahead. J Maxillofac Oral Surg. 2019;18(4):509-17.

31. 3Rose PT. Hair restoration surgery: Challenges and solutions. Clin Cosmet Investig Dermatol. 2015;8:361-70.

32. Oberli MA, Schoellhammer CM, Langer R, Blankschtein D. Ultrasound-enhanced transdermal delivery: Recent advances and future challenges. Ther Deliv. 2014;5(7):843-57.

33. Liao A, Lin $\mathrm{K}$, Chuang $\mathrm{H}$, Tsai $\mathrm{C}$, Lin $\mathrm{Y}$. ultrasound-mediated microbubble cavitation for transdermal minoxidil delivery and hair growth enhancement. 2020;1-12.

34. Kim JH, Na J, Bak DH, Lee BC, Lee E, Choi MJ, et al. Development of finasteride polymer microspheres for systemic application in androgenic alopecia. Int J Mol Med. 2019;43(6):2409-19.

Cite this article as: Chhetri DD, Huang X.

Management of androgenetic alopecia: a review. Int J Res Dermatol 2021;7:321-6. 